\title{
Letrozole supplementation during controlled ovarian stimulation in expected high responders: a pilot randomized controlled study
}

Xiaoyi Yang ${ }^{1,2,3}$, Ge Lin ${ }^{1,2,3}$, Guangxiu Lu ${ }^{1,2,3}$ and Fei Gong ${ }^{1,2,3^{*}}$

\begin{abstract}
Background: Almost all of the previous studies related with co-administration of letrozole in IVF cycles were performed in poor responders and letrozole may reduce the total gonadotropin dose required for ovarian stimulation, and the pregnancy rate did not decrease in poor responders. This study aimed to assess whether high responders co-treatment with letrozole reduced supraphysiological late follicular phase estradiol levels and the incidence of premature progesterone elevated at the end of the follicular phase, thereby impacting positively on endometrial receptivity.
\end{abstract}

Methods: A randomized parallel controlled study in a university-affiliated center include 130 high responders between October 2015 and August 2016. The patients were randomized on the first stimulation day of the IVF cycle and from stimulation day 5 receive letrozole (group A) or without letrozole treatment (group B).

Results: Although estradiol levels were significantly lower in the letrozole group (group $A)(P<0.001)$, progesterone elevation ( $>1.5 \mathrm{ng} / \mathrm{mL}$ was considered as a rise) on the day of $\mathrm{hCG}$ triggering $(15.4,7.7 \%)$ was not statistically significant $(P=0.170)$. RecFSH, the recovery rate of eggs, the high-quality embryo rate, and the thickness of endometrium ( $P=0.776$ ) were similar between the letrozole group(group $A)$ and control groups (group B). Clinical pregnancy rates were $53.1 \%(26 / 49)$ and $72.9 \%(35 / 48)$ in the letrozole and control groups, respectively, with a statistical significance $(P=0.043)$. Live birth rates were $42.9 \%(21 / 49)$ and $62.5 \%(30 / 48)$,showed a marginally significant difference $(P=0.053)$. The miscarriage rate did not significantly differ between the two groups.

Conclusions: In this pilot study, letrozole supplementation could not reduce the incidence of premature progesterone rise during the late follicular phase in stimulated in vitro fertilization cycles in expected high responders, producing a harmful effect on the pregnancy outcome.

Trial registration: China Clinical Trial Registration Center: ChiCTR-IPR-15006211 URL of the trial registry record: http://www.chictr.org.cn/showproj.aspx?.proj=10731. Trial registration date: 8 April, 2015. Date of first patient's enrolment: 5 October, 2015.

Keywords: Letrozole, Progesterone, Randomized study, High responders, In vitro fertilization

\footnotetext{
* Correspondence: gongfeizxxy@126.com

${ }^{1}$ Institute of Reproducitve and Stem Cell Engineering, Basic Medicine College,

Central South University, Changsha 410078, China

${ }^{2}$ Reproductive and Genetic Hospital of CITIC-XIANGYA, Changsha, China

Full list of author information is available at the end of the article
}

(c) The Author(s). 2019 Open Access This article is distributed under the terms of the Creative Commons Attribution 4.0 International License (http://creativecommons.org/licenses/by/4.0/), which permits unrestricted use, distribution, and reproduction in any medium, provided you give appropriate credit to the original author(s) and the source, provide a link to the Creative Commons license, and indicate if changes were made. The Creative Commons Public Domain Dedication waiver (http://creativecommons.org/publicdomain/zero/1.0/) applies to the data made available in this article, unless otherwise stated. 


\section{Background}

The effects of gonadotropin ovarian stimulation is a core component of in vitro fertilization (IVF) treatment. It was demonstrated that this might in part reflect the detrimental effects of gonadotropin ovarian stimulation on oocyte, embryo, and endometrial quality [1-3]. The impact of supraphysiological progesterone levels on endometrial maturation and receptivity is still a major concern in assisted reproduction. Increased serum progesterone levels in the late follicular phase are associated with reduced implantation rate [4]. Whether it is gonadotropin-releasing hormone $(\mathrm{GnRH})$ agonist or GnRH antagonist used for pituitary downregulation, the progesterone levels of more than $1.5 \mathrm{ng} / \mathrm{mL}$ on the day of human chorionic gonadotropin (hCG) administration in IVF-stimulated cycles were associated with lower pregnancy rates [5-7].

High estradiol (E2) levels have been implicated as another causative factor. Recent studies have shown that patients with high E2 levels in the late follicular phase have significant progesterone elevation $[8,9]$. Analysis of more than 60,000 cycles showed that serum E2 levels and the number of mature oocytes collected on the day of hCG administration also showed a statistical relationship with elevated progesterone and was associated with a lower pregnancy rate [10].

This mechanism might be related to the high reactivity of the ovary in the IVF stimulation cycle. An increased number of mature follicles and granulosa cells are present in high responders. It is suggested that a mechanism might exist to increase the progesterone levels associated with high ovarian responses during controlled ovarian stimulation. This idea was confirmed [11, 12], indicating that the incidence of progesterone elevation on the day of hCG administration was related to the ovarian response.

Milder stimulation regimens have been advocated as a means of mitigating these effects [13], but a recent study showed that the E2 levels were less affected by late-start mild protocols [14]. The question, therefore, remains whether medications of ovarian stimulation can ameliorate the negative effects on endometrial receptivity. It was proposed that this might be achieved using aromatase inhibitors (AIs), which inhibit the intraovarian aromatization of androgens to estrogens. Combining less gonadotropin stimulation to produce adequate numbers of oocytes with a simple oral adjuvant therapy aimed at preventing excessive E2 and progesterone levels, the resultant stimulation protocol might provide the "best of both worlds" solution, in which adequate numbers of oocytes were obtained, but not at the cost of detrimental impacts on oocytes, embryos, or endometrial quality. AIs have an established role in preventing the recurrence of estrogen-dependent tumors such as breast cancer [15]. The safety of long-term AIs has been repeatedly demonstrated in women conceiving after years of AI treatment [16].

It was widely used for poor responders because of lower total dosage and shorter duration [17, 18]. However, it was not routinely used for high responders in IVF stimulation cycles. So far, few studies have investigated its effect on the progesterone levels.

This study aimed to assess whether co-treatment with letrozole reduced supraphysiological late follicular phase E2 levels and the incidence of premature progesterone elevated at the end of the follicular phase, thereby impacting positively on endometrial receptivity.

\section{Methods}

\section{Patients}

This was a single-center randomized parallel controlled study in university-affiliated tertiary hospital reproductive center in Changsha, China. In this study, principles outlined inthe Declaration of Helsinki were followed and informed consent of all patientswas obtained.

The recruited participants were randomly assigned to two groups in a 1:1 ratio.

The Institutional Review Boards at Ethics Committee of Reproductive and Genetic Hospital CITIC-Xiangya approved the agreement. All participants signed the written informed consent. They participated in one study only and did not receive any funding for their participation in the study.

\section{Participants}

Altogether, 130 women undergoing IVF-embryo transfer (ET) treatment were enrolled. High responders were defined as those with an antral follicle count (AFC) of at least 15.The inclusion criteria were as follows: (1) medical indication for IVF treatment; (2) AFC between 15 and 23; (3) age 21-35 years; (4) at the first or the second treatment cycle; and (5) the body mass index (BMI) 18-28. The exclusion criteria were as follows: (1) medical contraindication to IVF treatment $(<4$ oocytes obtained); (2) previous documented poor response to ovary in IVF-stimulated cycles; (3) presence of endometriosis disease or uterine malformations; and (4) a history of unexplained miscarriages.

\section{Randomization}

On the first day of the IVF cycle, eligible patients were recruited and subjected to ultrasound scans. They were then randomly divided into two groups based on the sealed computer-generated list: letrozole and control groups. Random number list was generated by research 
department staff of the hospital and provided to the study nurse in a sealed envelope. After the patients were recruited, the assignment order was hidden until the research nurse specified the intervention. Physicians and embryologists were blinded to the assignments.

\section{Treatment}

All participants were subcutaneously administered 0.05 $\mathrm{mg}$ diphereline acetate (Diphereline $0.1 \mathrm{mg}$, Ipsen) daily from midluteal phase of the preceding IVF-ET cycle to hCG administration day with a standard short-acting GnRH-agonist protocol, and the initial dose of gonadotropin was determined based on the characteristics of each patient (age, BMI, and AFC).

In the letrozole group(group $\mathrm{A}, n=65$ ), the participants received the standard $\mathrm{GnRH}$-agonist protocol, which was daily intramuscular injections of 100-225 IU recFSH from stimulation day 1 (gonafal; Merck, Germany) until the day of hCG administration and co-treatment with letrozole $2.5 \mathrm{mg}$ (Jiangsu, Hengrui, China) daily from stimulation day 5 until the day of hCG administration. In the control group (group B, $n=65), 100-225 \mathrm{IU}$ of recFSH was injected intramuscularly daily from day 1 of stimulation (gonafal; Merck, Germany) to the day of hCG administration. A standard luteal phase support with progesterone was provided. Crinone 8\% (Progesterone Vaginal Gel) was initiated following retrieval by all 155 patients.

From the first day of the IVF cycle, ovarian responses were monitored by continuous vaginal scans and hormone measurements. hCG at a dose of 6500 IU (Merck) was given when at least three follicles were $18-20 \mathrm{~mm}$ in diameter. Oocyte retrieval was $35-36 \mathrm{~h}$ after the hCG injection.

\section{Outcome}

The primary end point of the study was as follows: The percentage of patients had progesterone elevation on the day of hCG triggering $(\geq 1.5 \mathrm{ng} / \mathrm{mL}$ was defined as elevation).

The secondary outcomes were as follows: (1) Serum E2 and progesterone levels on the hCG administration day (or the day before) and day 6 after ET; (2) Number of follicles $>12 \mathrm{~mm}$ on the day of hCG administration (or the day before) and oocytes obtained; and (3) Total IU of FSH used per cycle. (4) Clinical pregnancy rate.

Ultrasound-guided oocytes retrieved were performed with a $6.5-\mathrm{MHz}$ vaginal probe and a 16-gauge single-channel needle (Cook IVF, Cook, Australia). The recovered oocytes were inseminated according to the conventional IVF/intracytoplasmic sperm injection. After 16-18 h, they were checked for fertilization. When two pronuclei were visible, normal fertilization was considered. One or two normal embryos were transferred into the uterine cavity 3 days after removal. Blood was collected within 6 days after hCG administration to measure serum E2 and progesterone concentrations. The serum E2 and progesterone concentrations were rechecked 11 days after hCG administration.

Tables 1 and 2 summarize the baseline characteristics of the patients, clinical parameters from ovarian stimulation to embryo transfer, serum progesterone levels and clinical pregnancy rate.

Serum pregnancy test was performed 19 days after hCG administration. Pregnancies were diagnosed by an increasing concentration more than $200 \mathrm{IU} / \mathrm{mL}$ of serum $\beta$-hCG 14 days after ET. Clinical pregnancies were defined as ultrasound imaging of one or more gestational sacs or clear clinical signs of pregnancy.

First-trimester miscarriage was defined as a loss of clinical pregnancy before 70 days after ET. Abortion during late pregnancy was defined as the loss of clinical pregnancy after 70 days after ET. Live birth was defined as the birth of one or two living healthy children.

\section{Statistical analysis}

It was hard to define sample size based on all of the previous studies, because the data about AIs in high responders were quite limited in assisted reproductive technology cycles. Some studies found a difference of elevated $P>1.5 \mathrm{ng} / \mathrm{mL}$ increased with the ovarian response with $1-5,6-9,10-13,14-18$, and $>18$ oocytes $(4.5,3.9,8.3,12.1$, and $19.0 \%)$. Based on these reports, we supposed that the incidence of high serum progesterone levels on the day of hCG administration from 15 to $2 \%$ [11, 19], with $80 \%$ power at an $\alpha$ of $0.05,57$ patients were required per arm. Taking into account a drop-out rate of $15 \%, 65$ patients were required.

Quantitative indicators subject to normal distribution were presented as mean \pm standard deviation. The $P$ values were calculated using independent-sample $t$ tests. Median (Min, Max) was not used to describe the normal distribution, and $P$ value was calculated using the nonparametric test. The categorical indicators were described using frequency, and two independent samples were compared using the chi-square test or Fisher's exact test.Social Sciences version 22.0 was used. A $P$ value of $<0.05$ was considered statistically significant.

\section{Results}

This study was approved by the Ethics Committee of Reproductive and Genetic Hospital CITIC-Xiangya on November 20, 2014 (LL-SC-SG-2014-014) and registered in Changsha (ChiCTR-IPR-15006211) on April 8, 2015.The study was started on October 2015. The first subject was enrolled on October 5, 2015, and the last one on August 9, 2016. 
The flowchart of this trail is shown in Fig. 1.

The number of patients who withdrew from the study was 5 out of $65(7.7 \%)$ in the Group A and 1 out of 65 (1.5\%) in the Group B (Fig. 1).

Reasons for withdrawal were as follows: refusing to participate $(n=4)$ and joint pain $(n=1)$ in Group A (the study group) and contamination $(n=1)$ in Group $B$ (the control group).

No significant differences were found in the age of the women, duration of infertility, BMI, types of infertility, AFC, or baseline hormonal profile (FSH) between the two groups (Table 1).

\section{Primary outcomes}

The patients had progesterone elevation (a level more than $1.5 \mathrm{ng} / \mathrm{mL}$ was considered as PE) on the day of hCG triggering (15.4, 7.7\%), which was not statistically significant $(P=0.170)$.

\section{Secondary outcomes}

Progesterone levels were $1.03(0.8,1.26)$ and $0.91(0.71$, $1.15) \mathrm{ng} / \mathrm{mL}(P<0.001)$ on the day of hCG administration, while serum E2 levels in letrozole group were significantly lower than those in the control group; progesterone/E2 $(P<0.001)$ increased. Serum E2 and progesterone levels on the day before hCG administration and on day 6 after ET were similar between the letrozole and control groups.

RecFSH, the recovery rate of eggs, the fertilization rate, the high-quality embryo rate, and the thickness of endometrium were similar between the letrozole and control groups. No difference was found in the total IU of FSH used per cycle $(P=0.482)$.

Clinical pregnancy rates were $53.1 \%(26 / 49)$ and $72.9 \%$ (35/48) in the letrozole and control groups, respectively, which were statistically significant $(P=0.043)$. Live birth rates were $42.9 \%(21 / 49)$ and $62.5 \%(30 / 48)$ in the letrozole and control groups. $(P=0.053)$. Live birth rates were $42.9 \%(21 / 49)$ and $62.5 \%(30 / 48)$, showed a marginally significant difference $(P=0.053)$.The miscarriage rate did not significantly differ between the two groups (Table 2).

\section{Discussion}

This study revealed that letrozole supplementation could not reduce the incidence of premature progesterone rise/elevation $(P>1.5 \mathrm{ng} / \mathrm{mL})$ in the late follicular phase in stimulated IVF cycles in high responders.

Although progesterone had a key role in the IVF stimulation cycle and was particularly important for endometrial receptivity, the harmful effects of its serum level in the late follicular phase have been controversial and a cause of concern [20-22].

The effect of letrozole has been controversial in stimulated IVF cycles. Especially for poor responders, although the pregnancy rate did not increase significantly, the cancellation rate [23] and the miscarriage rate [16]decreased in the IVF-stimulated cycle with letrozole. When given to infertile women, decreased levels of estrogen led to increased FSH secretion from the pituitary gland. This increased FSH stimulated follicular development and was the basis of AI for ovulation induction

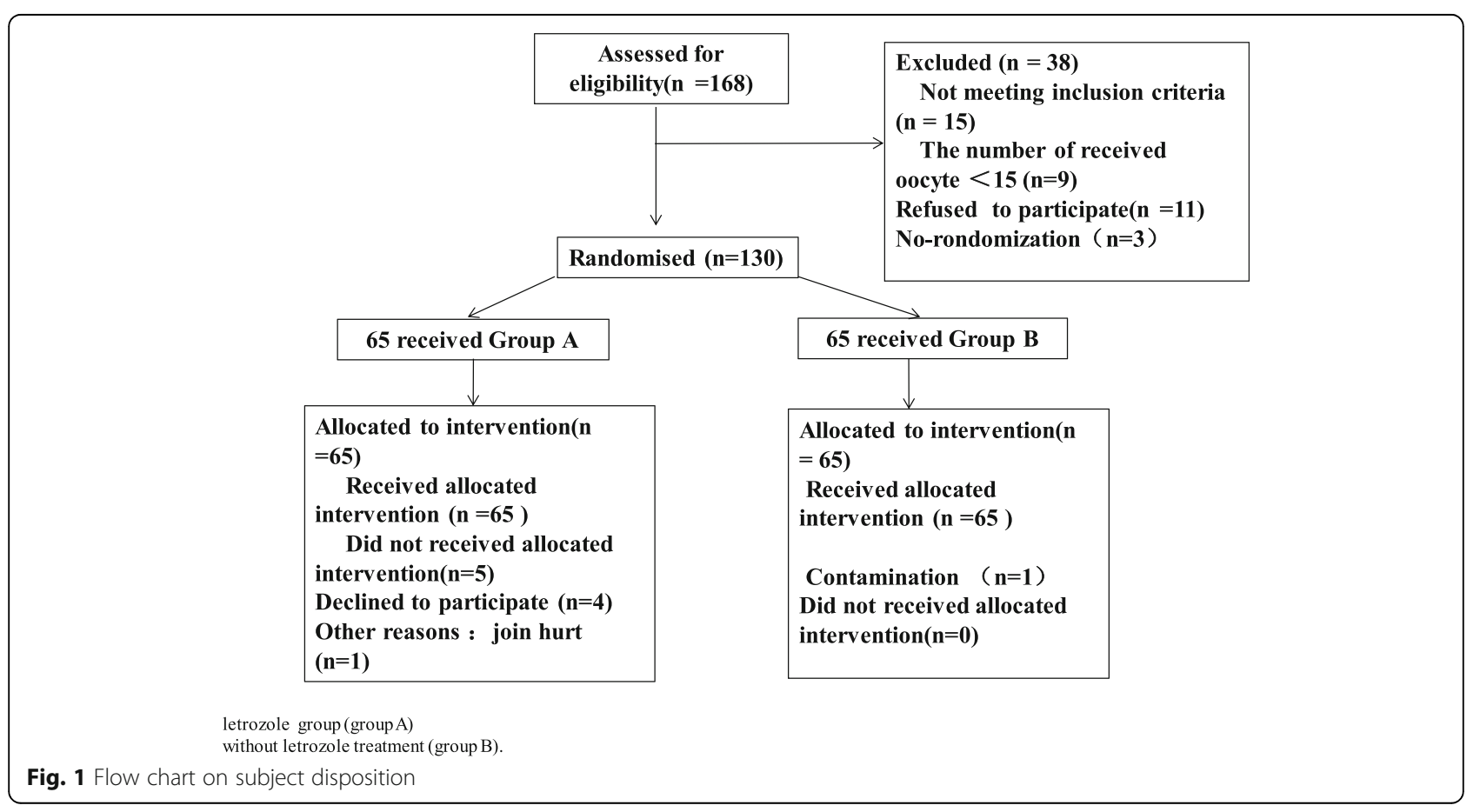


Table 1 Baseline characteristics of patients

\begin{tabular}{|c|c|c|c|c|}
\hline & Letrozole & No letrozole & Wilcoxon $W / t / X^{2}$ & $P$ \\
\hline$N$ & 65 & 65 & & \\
\hline Age of women (year) & $28(21,35)$ & $29(23,36)$ & -1.182 & 0.237 \\
\hline Body mass index $\left(\mathrm{kg} / \mathrm{m}^{2}\right)$ & $21.76 \pm 2.4$ & $22.13 \pm 2.45$ & -0.878 & 0.382 \\
\hline Duration of infertility (year) & $3(1,10)$ & $3(1,12)$ & -0.262 & 0.793 \\
\hline Number of embryo transfer & $2(0,2)$ & $2(0,2)$ & -0.229 & 0.819 \\
\hline Infertility type & & & 0.281 & 0.596 \\
\hline Primary infertility & $30(46.15 \%)$ & $27(41.54 \%)$ & & \\
\hline Secondary infertility & $35(53.85 \%)$ & $38(58.46 \%)$ & & \\
\hline Antrol follicle count & $22(14,23)$ & $22(12,30)$ & -0.592 & 0.554 \\
\hline Mean baseline hormone values (Day3) FSH (UI/L) & $5.36(2.72,11.27)$ & $5.51(3.24,12.01)$ & -0.17 & 0.865 \\
\hline Progesterone (nmol/L) & $0.27(0.1,0.95)$ & $0.27(0.1,1.07)$ & -0.142 & 0.887 \\
\hline
\end{tabular}

Table 2 Clinical/oocyte/embryo parameters from ovarian stimulation to embryo transfer relationship between serum progesterone levels and clinical pregnancy rates

\begin{tabular}{|c|c|c|c|c|}
\hline & Group A & Group B & Wilcoxon $W / t / X^{2}$ & $P$ \\
\hline Patients with P elevation on day of administraition day $\mathrm{n}(\%)$ & $15.4 \%(10 / 65)$ & $7.7 \%(5 / 65)$ & 1.884 & 0.170 \\
\hline RecFSH & $1275(0,2700)$ & $1350(787.52175)$ & -0.703 & 0.482 \\
\hline RecFSH/day & $137.5(0,225)$ & $142.5(98.44,197.73)$ & -1.735 & 0.083 \\
\hline Recovery rate of eggs & $13(1,28)$ & $12(3,34)$ & -1.516 & 0.129 \\
\hline Duration of GN(day) & $9(6,19)$ & $9(7,13)$ & -1.293 & 0.196 \\
\hline Fertilized egg number & $8.72 \pm 4.26$ & $8.63 \pm 4.15$ & 0.132 & 0.895 \\
\hline Fertilization rate & $69.2 \%(0,100 \%)$ & $78.5 \%(0,100 \%)$ & -1.493 & 0.135 \\
\hline Number of cleavage & $8.57 \pm 4.23$ & $8.53 \pm 4.19$ & 0.051 & 0.959 \\
\hline Cleavage rate & $100 \%(100,100 \%)$ & $100 \%(100,100 \%)$ & & \\
\hline High-quality embryo & $5(0,13)$ & $6(0,14)$ & -0.879 & 0.379 \\
\hline Rate of production of high-quality embryos & $60 \%(0,100 \%)$ & $70 \%(0,100 \%)$ & -1.21 & 0.226 \\
\hline $\mathrm{P}$ on the day of $\mathrm{hCG}$ administration $(\mathrm{ng} / \mathrm{mL})$ & $1.05(0.3,2.61)$ & $0.95(0.15,2.06)$ & -2.047 & 0.041 \\
\hline E2 on the day of hCG administration $(\mathrm{pg} / \mathrm{mL})$ & $1404.28(431.36227)$ & $2867(1043.62,7198)$ & -7.131 & $<0.001$ \\
\hline P/E2 on the day of hCG administration & $0.73(0.066,1.86)$ & $0.317(0.043,1.84)$ & -6.931 & $<0.001$ \\
\hline E2 on 1 day before the day of hCG administration & $546.95(0,2611)$ & $1102(0,5488)$ & -2.055 & 0.040 \\
\hline P on 1 day before the day of hCG administration & $0.545(0,1.65)$ & $0.52(0,1.83)$ & -0.33 & 0.741 \\
\hline P on 6th day post-ET (ng/mL) & $9.025(0,96.3)$ & $9.08(0,44.86)$ & -0.407 & 0.984 \\
\hline E2 on 6 th day post-ET (ng/mL) & $103.4(0,4737)$ & $127.75(0,2656)$ & -0.158 & 0.875 \\
\hline Thickness of endometrium & $12.1(9,17)$ & $12.2(8.3,17.8)$ & -0.284 & 0.776 \\
\hline Clinical pregnancy rate & $53.1 \%(26 / 49)$ & $72.9 \%(35 / 48)$ & 4.096 & 0.043 \\
\hline Implantation rate & $35.1 \%(33 / 94)$ & $47.4 \%(45 / 95)$ & 2.931 & 0.087 \\
\hline Incidence of OHSS & $0 \%(0 / 65)$ & $1.5 \%(1 / 65)$ & 1.008 & 1.000 \\
\hline Live birth rate & $42.9 \%(21 / 49)$ & $62.5 \%(30 / 48)$ & 3.752 & 0.053 \\
\hline First trimester miscarriage & $7.7 \%(2 / 26)$ & $5.7 \%(2 / 35)$ & 0.095 & 1.000 \\
\hline Second trimester miscarriage & $7.7 \%(2 / 26)$ & $5.7 \%(2 / 35)$ & 0.095 & 1.000 \\
\hline
\end{tabular}

Note: quantitative indicators subject to normal distribution were presented as mean \pm standard deviation. Median (Min, Max) was not used to describe the normal distribution. Group A: letrozole $2.5 \mathrm{mg} /$ day from cycle day 5 to the day before hCG administration. Group B: no letrozole; ET Embryo transfer, RecFSH Recombinant $\mathrm{FSH}, \mathrm{OHSS}$ Ovarian hyperstimulation syndrome 
[24, 25]. It is generally recommended to give letrozole for 5 days for ovulation induction or superovulation because its half-life is $45-72 \mathrm{~h}$. The main side effects include a mild headache and muscle or joint pain [26, 27]. However, in a late large RCT study in N Engl J Med shows, ovarian stimulation with letrozole in women with unexplained infertility resulted in a lower frequency of live birth [28].

In the present study, no difference was found in the incidence of progesterone elevation in the late follicular phase in patients treated with the GnRH-agonist protocol. Previous randomized trials of $\mathrm{GnRH}$ agonists co-treated with letrozole did not measure the late follicular progesterone levels.

Almost all of the previous studies related with co-administration of letrozole in IVF cycles were performed in poor responders $[29,30]$. The data about AIs in high responders were quite limited in assisted reproductive technology cycles. In a pilot study, letrozole stimulation from endometrial preparations in PCOS patients in frozen embryo transfer cycles may have better results than hormone manipulation or hMG stimulation. [28]. To date, no studies have reported the use of letrozole to specifically improve clinical outcome for high responders in IVF stimulation cycles(for example: progesterone levels). In this study, in addition to lower serum E2 levels, a little difference was found in the levels of other hormones that responded to high-response co-treatment with letrozole compared with the patients who did not take it.

Progesterone in the early follicular phase has an adrenal origin $[31,32]$. In a recent study, decreasing progesterone levels in early follicular phase seems to be beneficial for cumulative live birth rates. However, in the late follicular phase, progesterone is mainly derived from mature follicles. Higher daily FSH dose was the factor most related to the occurrence of serum progesterone elevation $[6,10,33]$. The meta-analysis of Papanikolao $[5,32]$ showed that AI administration induced an acute hypoestrogenic state that released the hypothalamic-pituitary axis from estrogenic negative feedback, in turn increasing FSH secretion and ovarian follicle development [27]. Letrozole appeared to have the potential to reduce the total gonadotropin dose required for ovarian stimulation. In this study, recFSH was similar between the letrozole and control groups. The patients had progesterone elevation on the day of hCG administration, which was not statistically significant. More eggs were recovered in the letrozole groups. Considering the fact that high progesterone levels were positively related to the number of mature follicles, it was possible that low responders with a lower expression of FSH receptor in the granulosa cells were demonstrated [33]. Contrary to this situation, high responders showed the overexpression of FSH receptors in their follicular granulosa cell population [34, 35] and might be the reason why letrozole could not reduce the total gonadotropin dose needed for ovarian stimulation in hyper-responders.

Two successive enzymatic reactions convert progesterone into androgen (by 17ahydroxylase and C17, 20 lyase) and then again into estrogen by aromatase [36, 37]. In our study, add to letrozole means aromatase enzyme is blocked, and it may lead to accumulation of such as progesterone, testosterone, and $17 \alpha$-progesterone on progesterone levels.

This study suggested that co-treatment with letrozole in high responders for agonist protocols could not reduce the incidence of premature progesterone increase in the late follicular phase in stimulated IVF cycles, producing a harmful effect on the pregnancy outcome. Therefore, this study did not continue to expand.

This study found that even higher levels of progesterone in high responders might have a negative impact on the implantation rate of high-quality cleavage-stage embryos [38]. Therefore, the question was what should be done in patients with advanced ovarian progesterone levels. An alternative means of reducing the exposure of gametes and endometrium to supraphysiological levels of progesterone was to selectively freeze, thaw, and transfer all embryos into a nonstimulated cycle. This option of "compartmentalizing" IVF treatment attracted increasing attention as recently published data suggested that clinical outcomes after embryo vitrification were satisfactory. However, in China, most patients prefer to choose fresh-cycle transplantation instead of cryopreservation for economic reasons and psychological factors. The question, therefore, remains whether medications for ovarian stimulation can ameliorate the negative effects on endometrial receptivity. The limitations of this pilot study were the use of high responders in GnRH-agonist cycles and the small sample size. The definition of high responder is just based on antral follicle counts at baseline. A further high-quality study is required to validate the findings. Moreover, the administration of letrozole offered a new insight to high-responders.

\section{Conclusions}

Therefore, the fact that co-treatment with letrozole in high responders cannot be a positive factor for the IVF outcome must be considered seriously.The effect of elevated progesterone levels in high responders on clinical outcomes might be due to a balance between the two antagonistic factors: the possibility of obtaining more eggs and ovarian response and the endometrial preterm birth-impaired possibility to progesterone. 


\section{Abbreviations}

AFC: qAntral follicle count; Als: Aromatase inhibitors; BMl: body mass index; E2: Estradiol; ET: Embryo transfer; FET: Frozen embryo transfer; FSH: Follicle stimulating hormone; GnRH: Gonadotropingonadotropin-releasing hormone; HCG: Human chorionic; IVF: In vitro fertilization; OHSS: Ovarian hyperstimulation; P: progesterone

\section{Acknowledgements}

The authors thanks all the patients and staff at the Reproductive and Genetic Hospital of CITIC-XIANGYA and Professor Macklon N for his idea.

\section{Funding}

None.

\section{Availability of data and materials}

The datasets supporting the conclusions of this article are included within the article.

\section{Authors' contributions}

XYY implementation of the study, data acquisition, analysis and interpretation, and drafting of the manuscript; GL: data interpretation, seeking ethical approval. GXL and FG study design and writing the manuscript. All authors provided final approval of the submitted manuscript.

\section{Ethics approval and consent to participate}

This study was approved by the Ethics Committee of Reproductive and Genetic Hospital CITIC-Xiangya on November 20, 2014 (LL-SC-SG-2014-014).

\section{Consent for publication}

Written informed consent for publication was obtained from all participants.

\section{Competing interests}

The authors declared that they have no conflicts of interest to this work.

\section{Publisher's Note}

Springer Nature remains neutral with regard to jurisdictional claims in published maps and institutional affiliations.

\section{Author details}

${ }^{1}$ Institute of Reproducitve and Stem Cell Engineering, Basic Medicine College, Central South University, Changsha 410078, China. ${ }^{2}$ Reproductive and Genetic Hospital of CITIC-XIANGYA, Changsha, China. ${ }^{3}$ Key Laboratory of Reproductive and Stem Cell Engineering, National Health and Family Planning Commission, Changsha, China.

\section{Received: 7 December 2018 Accepted: 12 April 2019}

\section{Published online: 10 May 2019}

\section{References}

1. Macklon NS, et al. The science behind 25 years of ovarian stimulation for in vitro fertilization. Endocr Rev. 2006;27(2):170.

2. Rubio C, et al. Prospective cohort study in high responder oocyte donors using two hormonal stimulation protocols: impact on embryo aneuploidy and development. Hum Reprod. 2010;25(9):2290.

3. Baart EB, et al. Milder ovarian stimulation for in-vitro fertilization reduces aneuploidy in the human preimplantation embryo: a randomized controlled trial. Hum Reprod. 2007:22(4):980-8.

4. Venetis CA, et al. Is progesterone elevation on the day of human chorionic gonadotrophin administration associated with the probability of pregnancy in in vitro fertilization? A systematic review and meta-analysis. Hum Reprod Update. 2007:13(4):343-55.

5. Papanikolaou EG, et al. Aromatase inhibitors in stimulated IVF cycles. Reprod Biol Endocrinol. 2011;9(1):1-4.

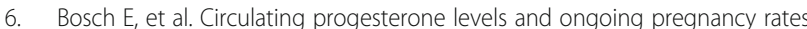
in controlled ovarian stimulation cycles for in vitro fertilization: analysis of over 4000 cycles. Hum Reprod. 2010;66(8):2092-100.

7. Santosribeiro $S$, et al. Live birth rates after IVF are reduced by both low and high progesterone levels on the day of human chorionic gonadotrophin administration. Hum Reprod. 2014;29(8):1698-705.
8. Kyrou D, et al. Does the estradiol level on the day of human chorionic gonadotrophin administration have an impact on pregnancy rates in patients treated with rec-FSH/GnRH antagonist? Hum Reprod. 2009;24(11): 2902-9.

9. Kyrou D, et al. The relationship of premature progesterone rise with serum estradiol levels and number of follicles in GnRH antagonist/recombinant FSH-stimulated cycles. Eur J Obstet Gynecol Reprod Biol. 2012;162(2):165-8.

10. Venetis CA, et al. Progesterone elevation and probability of pregnancy after IVF: a systematic review and meta-analysis of over 60000 cycles. Hum Reprod Update. 2013;19(5):433-57.

11. Griesinger $\mathrm{G}$, et al. Progesterone elevation does not compromise pregnancy rates in high responders: a pooled analysis of in vitro fertilization patients treated with recombinant follicle-stimulating hormone/gonadotropinreleasing hormone antagonist in six trials. Fertil Steril. 2013;100(6):1622.

12. Racca $A$, et al. Impact of late-follicular phase elevated serum progesterone on cumulative live birth rates: is there a deleterious effect on embryo quality? Hum Reprod. 2018.

13. Fauser BC, et al. Mild ovarian stimulation for IVF: 10 years later. Hum Reprod. 2010;25(11):2678

14. Blockeel C, et al. Follicular phase endocrine characteristics during ovarian stimulation and GnRH antagonist cotreatment for IVF: RCT comparing recFSH initiated on cycle day 2 or 5. J Clin Endocrinol Metab. 2011;96(4):1122-8.

15. Finn RS, et al. Palbociclib and Letrozole in advanced breast Cancer. N Engl J Med. 2016;375(20):1925.

16. Tatsumi T, et al. No increased risk of major congenital anomalies or adverse pregnancy or neonatal outcomes following letrozole use in assisted reproductive technology. Hum Reprod. 2016;32(1):125.

17. Garciavelasco JA, et al. The aromatase inhibitor letrozole increases the concentration of intraovarian androgens and improves in vitro fertilization outcome in low responder patients: a pilot study. Dkgest of the World Latest Medical Information. Fertil Steril. 2005:84(1):82-7.

18. Goswami SK, et al. A randomized single-blind controlled trial of letrozole as a low-cost IVF protocol in women with poor ovarian response: a preliminary report. Hum Reprod. 2004;19(9):2031-5.

19. Bosch E. Does progesterone elevation compromise pregnancy rates in high responders? Insufficient evidence to draw a conclusion. Fertil Steril. 2014;101(1):e3-4

20. Bourgain C, Devroey P. The endometrium in stimulated cycles for IVF. Hum Reprod Update. 2003;9(6):515-22.

21. $\mathrm{Xu} B$, et al. Serum progesterone level effects on the outcome of in vitro fertilization in patients with different ovarian response: an analysis of more than 10,000 cycles. Fertil Steril. 2012;97(6):1321-1327.e4.

22. Requena A, et al. High progesterone levels in women with high ovarian response do not affect clinical outcomes: a retrospective cohort study. Reprod Biol Endocrinol Rb \& E. 2014;12(1):69.

23. Ozmen B, et al. Use of aromatase inhibitors in poor-responder patients receiving $\mathrm{GnRH}$ antagonist protocols $i$. Reprod BioMed Online. 2009:19(4):478-85.

24. Casper RF, Mitwally MFM. A historical perspective of aromatase inhibitors for ovulation induction. Fertil Steril. 2012:98(6):1352-5.

25. Casper RF, Mitwally MF. Review: aromatase inhibitors for ovulation induction. Clin Endocrinol. 2015;100(5):1742-7.

26. Ferrero $\mathrm{S}$, et al. Pharmacological treatment of endometriosis: experience with aromatase inhibitors. Drugs. 2009;69(8):943-52.

27. Requena $A$, et al. Use of letrozole in assisted reproduction: a systematic review and meta-analysis. Hum Reprod Update. 2008;14(6):571-82.

28. Diamond MP, et al. Letrozole, gonadotropin, or clomiphene for unexplained infertility. N Engl J Med. 2016;70(13):1230-40.

29. Ozcan CP, et al. The comparision of effect of microdose $\mathrm{GnRH}$-a flare-up, $\mathrm{GnRH}$ antagonist/aromatase inhibitor letrozole and GnRH antagonist/ clomiphene citrate protocols on IVF outcomes in poor responder patients. Gynecol Endocrinol. 2014;30(7):485-9.

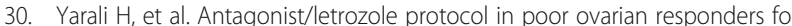
intracytoplasmic sperm injection: a comparative study with the microdose flare-up protocol. Fertil Steril. 2009:92(1):231-5.

31. Judd $\mathrm{S}$, et al. The source of pulsatile secretion of progesterone during the human follicular phase. J Clin Endocrinol Metab. 1992;74(2):299-305.

32. De GC, et al. Progesterone serum levels during the follicular phase of the menstrual cycle originate from the crosstalk between the ovaries and the adrenal cortex. Hum Reprod. 2002;17(4):933. 
33. Cai J, et al. Poor ovarian response to gonadotropin stimulation is associated with low expression of follicle-stimulating hormone receptor in granulosa cells. Fertil Steril. 2007;87(6):1350-6.

34. Bosch $\mathrm{E}$, et al. Premature luteinization during gonadotropin-releasing hormone antagonist cycles and its relationship with in vitro fertilization outcome. Fertil Steril. 2003;80(6):1444-9.

35. Haas J, et al. The VEGF and PEDF levels in the follicular fluid of patients CO- treated with LETROZOLE and gonadotropins during the stimulation cycle. Reprod Biol Endocrinol. 2018;16(1):54.

36. Eldaly $A A$, et al. Aromatase inhibitors for ovulation induction: John Wiley \& Sons, Ltd; 2006. p. 537-46.

37. Goldrat $\mathrm{O}$, et al. Progesterone levels in letrozole associated controlled ovarian stimulation for fertility preservation in breast cancer patients. Hum Reprod. 2015;30(9):2184-9.

38. Bosch, E., High responders and patients with a good prognosis are not immune to the negative impact on live birth rate of elevated $\mathrm{P}$ on the day of triggering. Fertil Steril, 2015. 103(6): p. 1423-1423.

Ready to submit your research? Choose BMC and benefit from:

- fast, convenient online submission

- thorough peer review by experienced researchers in your field

- rapid publication on acceptance

- support for research data, including large and complex data types

- gold Open Access which fosters wider collaboration and increased citations

- maximum visibility for your research: over $100 \mathrm{M}$ website views per year

At BMC, research is always in progress.

Learn more biomedcentral.com/submissions 